\title{
ASG Version of Integral Sliding Mode Robust Controller for AV Nonholonomic 2D Models Avoiding Obstacles
}

Hector Vargas ( $\sim$ hvargasa2000@alumno.ipn.mx )

IPN: Instituto Politecnico Nacional https://orcid.org/0000-0002-0310-6172

Jesús Alberto Meda

Instituto Politécnico Nacional: Instituto Politecnico Nacional

Alexander Poznyak

CINVESTAV IPN: Centro de Investigacion y de Estudios Avanzados del Instituto Politecnico Nacional

\section{Research Article}

Keywords: Car obstacle avoidance, nonholonomic dynamic model, integral sliding mode control, averaged subgradient method

Posted Date: April 12th, 2021

DOI: https://doi.org/10.21203/rs.3.rs-351427/v1

License: (a) (1) This work is licensed under a Creative Commons Attribution 4.0 International License. Read Full License

Version of Record: A version of this preprint was published at Nonlinear Dynamics on April 21st, 2022. See the published version at https://doi.org/10.1007/s11071-022-07408-4. 


\title{
ASG Version of Integral Sliding Mode Robust Controller for AV Nonholonomic 2D Models Avoiding Obstacles
}

\author{
Hector Vargas · Jesús A. Meda - Alexander Poznyak
}

Received: date / Accepted: date

\begin{abstract}
In this work, a new robust controller is designed and analysed providing an autonomous vehicle, moving within a 2D - plain, with the ability to avoid collision with a set of obstacles despite of the presence of uncertainties in the Autonomous Vehicle (AV) with nonholonomic dynamic. The state variables (2 plain coordinates and 3 angles) and their velocities are assumed to be measurable. The controller design is based on Integral Sliding Mode (ISM) concept, aimed to minimize a given convex (not obligatory strongly convex) function of the current state. The subgradient of this cost function is also supposed to be measurable online. An optimization type algorithm is developed and analyzed using ideas of the Averaged Subgradient (ASG) technique. The main results consist in proving the reachability of the desired regime (non stationary analogue of sliding surface) from the beginning of the process and obtaining an explicit upper bound for the cost function decrement, that is, a functional convergence is proven and the rate of convergence is estimated, providing multiple obstacle avoidance. A numerical example depicts a good performance of the suggested hereby method.
\end{abstract}

Keywords Car obstacle avoidance $\cdot$ nonholonomic dynamic model - integral sliding mode control . averaged subgradient method

\section{H. Vargas}

SEPI-ESIME-ZAC, Instituto Politécnico Nacional, Mexico city, Mexico.

E-mail: hvargasa2000@alumno.ipn.mx

J.A. Meda

SEPI-ESIME-ZAC, Instituto Politécnico Nacional, Mexico city, Mexico.

E-mail: jmedac@ipn.mx

A. Poznyak

CINVESTAV-IPN, Mexico city, Mexico.

E-mail: apoznyak@ctrl.cinvestav.mx

\section{Introduction}

\subsection{Brief Survey}

Autonomous vehicles has gained great interest within the engineering community due to its wide applications such as industrial, or military, among others. The plenty methods for modelling, and controlling AV have been developed in recent years. As a result of such research the concept of obstacles avoidance has inevitably appeared along with a wide variety of approaches. Artificial vision and intelligent algorithms have been widely used to obtain the position of an $\mathrm{AV}$, and to pose it in a desired position: such tools enable the AV with the ability to identify prohibit zones as well. These approaches are described in [1, 22, [3], [4], where they have succeed in solving the problem partially. On the other hand, there are techniques based on dynamics of the electromechanical system models, which require measuring their position and velocity throughout moment to be controlled. In addition, one needs to know the environment in which the controlled system performs that requires a nonlinear control application. On-line optimization, designed specially for a dynamic systems and containing uncertainties in their models, is one of the forms of robust control. In 5 studies the optimization problem with constraints for linear and time invariant dynamic systems (LTI), where the dimension of control actions is smaller than the system state vector. The problem is to design a control signal capable to generate the system dynamics, avoiding some prohibited regions and minimizing the suggested cost function. The finite time convergence to a neighborhood of the equilibrium point is proven. In 6 an algorithm is developed which is based on the convex optimization concept applied to a dynamic plant modeled using the Lagrangian ap- 
proach, given by a differential standard equation of the second ordinary order and with unknown right-hand side, but with accessible states, as well as their speeds. Article [7] developed a finite-time optimal formation tracking for planar vehicles technique with holonomics dynamics, using Pontryagin's maximum principle on a Lie group. In 8 the trajectory planning and tracking control of the autonomous bicycle robot are considered and solved despite the nonholonomic dynamics. The desired motion trajectory of the contact point of the bicycle's rear wheel is constructed using a parameterized polynomial curve that can connect two given endpoints with associated tangent angles. The paper [9] works with a double-layer control framework is proposed to construct the coordinated lateral and longitudinal motion control for over-actuated autonomous electric vehicles, which are independently driven by four in-wheel motors using sliding mode control. Paper 10 deals with a fixed-time stabilization problem for a kind of nonholonomic systems in chained form with unmatched uncertainties and time-varying output constraints. A tan-type barrier Lyapunov function is used to solve the problem that with time-varying output constraints imply. A state feedback controller is designed using a power integrator technique and switching control strategy. and finally in an adaptive control strategy for hypersonic flight vehicles (HFVs) subject to parametric uncertainties is introduced. To achieve this, the disturbed control-oriented model of HFVs with uncertain parameters and faulty actuators is first rewritten into a parameterized form. By introducing barrier Lyapunov functions to design procedures, specific tracking performances of velocity and altitude are guaranteed, while the other flight states of angle of attack, flight path angle, pitch angle and pitch angle rate can be kept within the prescribed ranges.

In this paper ISM approach together with the ASG technique (supposing that the current sub-gradient $\partial F\left(x_{t}\right)$ of the convex function $F\left(x_{t}\right)$ to be optimized is available on-line) is applied. The obstacles are included in the cost function as penalty terms. So with the specially selected parameters of the designed controller, the functional convergence of the convex (not obligatory strongly convex) loss function to its minimal value is proven.

\section{Main contribution}

1. A variation of the known Lagrangian with nonholonomic constrains dynamic model is derived and analysed.

2. A new concept of ASG version of ISM control technique is designed.
3. A complete analysis of the functional convergence of the controlled system to the desired dynamics is presented.

4. A high level of uncertainties (inertia tensor, forces non-potentials such as: friction, hysteresis or Coriolis) in the system model is admitted.

5. The effectiveness of the method is demonstrated theoretically and with a numerical simulation, where a car robot $(\mathrm{AV})$ avoids obstacles reaching a desired point.

\section{Paper structure}

The article is organized as follows: The main contributions are in 2. The problem formulation are introduced in section 3 and section 4 contains the full mathematical car robot model description. In section 5 , the main assumptions and the cost function definition mentioned, then the development of the so-called desired dynamics and its properties are in section 6 , where full mathematical proof is provided. In section 7 , the structure of the ISM robust controller is presented and the main theorem of this work is introduced and proven. Theoretical results are depicted by a numerical simulation in section 8, where a AV avoids several obstacles to reach a desired position. Finally, the conclusions are in section 9 .

\section{Nonholonomic AV models}

\subsection{Nonholonomic Euler-Lagrange models}

\subsubsection{Coordinate Systems}

Two different coordinate systems (frames) need to be defined (see Fig 1).

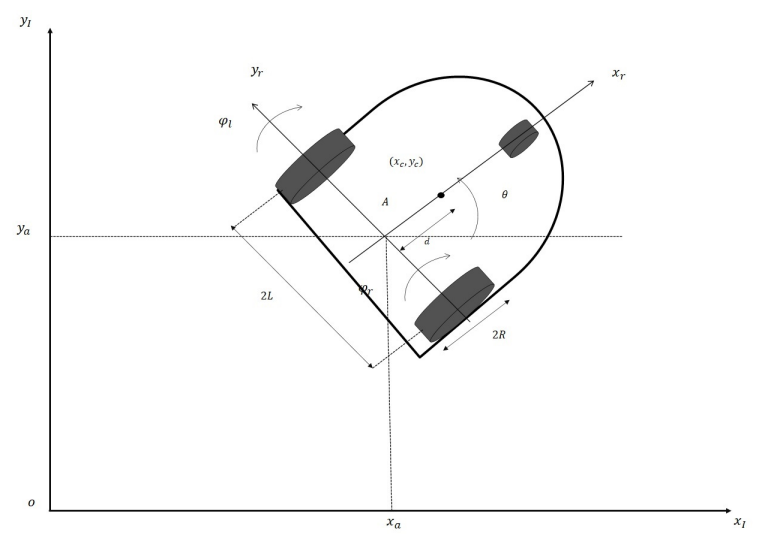

Fig. 1 AV description. 
1. Inertial Coordinate System: This coordinate system is a global frame which is fixed in the environment or plane in which the AV moves in. Moreover, this frame is considered as the reference frame and is denoted as $\left\{x_{I}, y_{I}\right\}$.

2. Relative (or proper) Coordinate System: This coordinate system is a local frame attached to the considered AV, and thus, moving with it. This frame is denoted as $\left\{x_{r}, y_{r}\right\}$. The origin of the relative frame is defined to be the mid-point $A$ on the axis between the wheels. The center of mass $C$ of the AV is assumed to be on the axis of symmetry, at a distance $d$ from the origin $A$.

As shown in Fig 1 the robot position and orientation in the Inertial Frame can be defined as

$q^{I}:=\left(x_{a}, y_{a}, \theta\right)^{\top}$

The important issue that needs to be explained at this stage is the mapping between these two frames 11. The position of any point on the AV can be defined in the inertial frame and the relative frame as follows:

$\Theta^{I}:=\left(x^{I}, y^{I}, \theta^{I}\right)^{\top}, \Theta^{r}:=\left(x^{r}, y^{r}, \theta^{r}\right)^{\top}$

Then, the two coordinates are related by the following transformation:

$\Theta^{I}=R(\theta) \Theta^{r}$

where $R(\theta)$ is the orthogonal $\left(R^{\boldsymbol{\top}}(\theta)=R^{-1}(\theta)\right)$ rotation matrix

$R(\theta)=\left[\begin{array}{ccc}\cos \theta & -\sin \theta & 0 \\ \sin \theta & \cos \theta & 0 \\ 0 & 0 & 1\end{array}\right]$

This transformation is enable also the handling of motion between frames.

\subsubsection{Kinematic constraints of the differential-drive mobil AV}

Here we will show that the motion of a differentialdrive mobile $\mathrm{AV}$ is characterized by two non-holonomic constraint equations, which are obtained by two main assumptions:

- No lateral slip motion: This constraint simply means that the mobil AV can move only in a curved motion (forward and backward) but not sideward. In the relative frame, this condition means that the velocity of the center-point $\mathrm{A}$ is zero along the lateral axis, namely, for any time $t \geq 0$

$y^{r}=0, \dot{y}^{r}=0$ which can be expressed as

$-\dot{x}_{a} \sin \theta+\dot{y}_{a} \cos \theta=0$

- Pure rolling constrain: The pure rolling constraint represents the fact that each wheel maintains a one contact point $p$ with the ground. There is no slipping of the wheel in its longitudinal axis $\left(x^{r}\right)$ and no skidding in its orthogonal axis $\left(y^{r}\right)$.

The velocities of the contact points in the relative frame are related to the right $(r)$ and left $(l)$ wheel velocities by:

$v_{p}=R \dot{\varphi}_{r}, v_{l}=R \dot{\varphi}_{l}$

In the inertial frame, these velocities can be calculated as a function of the velocities of the AV center-point $A$ :

$\left.\begin{array}{rl}\dot{x}_{p R} & =\dot{x}_{a}+L \theta \cos \theta \\ \dot{y}_{p R} & =\dot{y}_{a}+L \theta \sin \theta\end{array}\right\}$

and

$\left.\begin{array}{rl}\dot{x}_{p L} & =\dot{x}_{a}+L \dot{\theta} \cos \theta \\ \dot{y}_{p L} & =\dot{y}_{a}+L \dot{\theta} \sin \theta\end{array}\right\}$

Using the rotation matrix $R(\theta)$ (4) and (7), the rolling constraint equations (6) are formulated as follows:

$\left.\dot{x}_{p R} \cos \theta+\dot{y}_{p R} \sin \theta=R \dot{\varphi}_{R}\right\}$

$\left.\dot{x}_{p L} \cos \theta+\dot{y}_{p L} \sin \theta=R \dot{\varphi}_{L}\right\}$

Applying the contact points velocities equation (8) (9), and , the three constraint equations (6) and (10) can be rewritten in the following matrix form:

$\Lambda(q) \dot{q}=0$

where $q=\left(x_{a}, y_{a}, \theta, \varphi_{R}, \varphi_{L}\right)^{\top}$ is the generalized position vector and $\Lambda(q)$ is

$\Lambda(q)=:\left[\begin{array}{ccccc}-\sin \theta & \cos \theta & 0 & 0 & 0 \\ \cos \theta & \sin \theta & L & -R & 0 \\ \cos \theta & \sin \theta & -L & 0 & -R\end{array}\right]$

\subsubsection{Euler-Lagrange equations for nonholonomic dynamic models}

To derive equations of motion for nonholonomic systems (with constrains depending on the derivatives $\dot{q}$ of the generalized coordinate) we will apply the, socalled, Lagrangian approach using the Hamilton's principle and the Euler-Lagrange equations, respectively. In case when we have no constrains for dynamic trajectories, according to the Hamiltonian principle [12], [13]. 
the equations of motion for the considered system provides the extremal value for the Hamiltonian action, that is,

$$
\int_{t=a}^{b} L(q, \dot{q}, t) d t \rightarrow \operatorname{extr}_{q, \dot{q} \in \mathbb{R}^{n}}
$$

where $L=T-\Pi$ is the Lagrange function with the kinetic $T$ and potential $\Pi$ energies, respectively. This corresponds the condition

$\delta \int_{t=a}^{b} L(q, \dot{q}, t) d t=\int_{t=a}^{b}\left(\frac{\partial^{\top} L}{\partial q} \delta q+\frac{\partial^{\top} L}{\partial \dot{q}} \delta \dot{q}\right) d t=0$

Here we choose virtual (under fixed $t$ ) variations $\delta q_{t}$ of the curve $q_{t}(t \in[a, b])$ in such a way that $\delta q_{t=a}=$ $\delta q_{t=b}=0$. Integrating by parts with fixed endpoints we get

$\int_{t=a}^{b}\left(\frac{\partial^{\top} L}{\partial q}-\frac{d}{d t} \frac{\partial^{\top} L}{\partial \dot{q}}\right) \delta q d t=0$

which gives us, from the arbitrariness of $\delta q$, the EulerLagrange equation (in the vector form)

$\frac{\partial L}{\partial q}-\frac{d}{d t} \frac{\partial L}{\partial \dot{q}}=0$

Unfortunately, for the nonholonomic systems when there are constrains for the admissible trajectories we can conclude 15 since the admissible virtual variations $\delta q$ are not arbitrary. They should satisfy (11) for all $t \geq 0$, or equivalently,

$\Lambda(q) \delta q=0$

To apply correctly in our case the Hamiltonian principle we need to take into account the relation (11) considering the following extremal problem:

$$
\begin{gathered}
\int_{t=a}^{b} L(q, \dot{q}, t) d t \rightarrow \underset{q, \dot{q} \in \mathbb{R}^{n}}{\operatorname{extr}} \\
\text { under constrains }
\end{gathered}
$$

Using the Lagrange multipliers approach we may conclude that the variation of the Hamiltonian action (13) around the admissible extremal curve $q$, satisfying the constrains 16, corresponds to the condition (the extended version of D'Alembert's principle)

$\delta \int_{t=a}^{b} L(q, \dot{q}, t) d t+\int_{t=a}^{b} \lambda^{\top} \Lambda(q) \delta q d t=0$

where $\lambda$ is the vector of Lagrange multipliers depending on $t$ and the virtual variations now are arbitrary. Following the analogous procedure as before we conclude that (18) implies $\int_{t=a}^{b}\left(\frac{\partial^{\top} L}{\partial q} \delta q+\frac{\partial^{\top} L}{\partial \dot{q}} \delta \dot{q}+\lambda^{\top} \Lambda(q) \delta q\right) d t=0$

And again the integration by parts of the second term leads to

$\int_{t=a}^{b}\left(\frac{\partial^{\top} L}{\partial q}-\frac{d}{d t}\left(\frac{\partial^{\top} L}{\partial \dot{q}}\right)+\lambda^{\top} \Lambda(q)\right) \delta q d t=0$

which, by the arbitrariness of $\delta q$, is possible if and only if

$\frac{\partial L}{\partial q}-\frac{d}{d t} \frac{\partial L}{\partial \dot{q}}+\Lambda^{\top}(q) \lambda=0$

or equivalently,

$\frac{d}{d t} \frac{\partial L}{\partial \dot{q}}-\frac{\partial L}{\partial q}=\Lambda^{\top}(q) \lambda$

In the presence of external nonpotential forces $Q_{\text {nonpot }}$ the Euler-Lagrange equation for nonholonomic systems leads to

$\frac{d}{d t} \frac{\partial L}{\partial \dot{q}}-\frac{\partial L}{\partial q}=Q_{\text {nonpot }}+\Lambda^{\top}(q) \lambda$

The kinetic energy $T$ for the considered AV contains three parts:

- the kinetic energy of the AV platform

$T_{c}=\frac{1}{2} m_{c} v_{c}^{2}+\frac{1}{2} I_{c} \dot{\theta}^{2}$

- the kinetic energy of the right wheel

$T_{w R}=\frac{1}{2} m_{w} v_{w R}^{2}+\frac{1}{2} I_{m} \dot{\theta}^{2}+\frac{1}{2} I_{w} \dot{\varphi}_{R}^{2}$

- the kinetic energy of the left wheel

$T_{w L}=\frac{1}{2} m_{w} v_{w L}^{2}+\frac{1}{2} I_{m} \dot{\theta}^{2}+\frac{1}{2} I_{w} \dot{\varphi}_{L}^{2}$

where $m_{c}$ is the mass of the AV without the driving wheels and actuators (DC motors), $m_{w}$ is the mass of each driving wheel (with actuator), $I_{c}$ is the moment of inertia of the AV with respect to the vertical axis passing through the center of mass, $I_{w}$ is the moment of inertia of each driving wheel with a motor with respect to the wheel axis, and $I_{m}$ is the moment of inertia of each driving wheel with a motor with repect to the wheel diameter. Taking into account that all velocities may be expressed as a function of the generalized coordinates using the general velocity equation in the inertial frame, namely, as $v_{i}^{2}=\dot{x}_{i}^{2}+\dot{y}_{i}^{2}$, and in view of the relations

$$
\begin{gathered}
x_{c}=x_{a}+d \cos \theta, y_{c}=y_{a}+d \sin \theta \\
x_{w R}=x_{a}+L \sin \theta, y_{w R}=y_{a}+L \cos \theta \\
x_{w L}=x_{a}-L \sin \theta, y_{w L}=y_{a}+L \cos \theta
\end{gathered}
$$


we may conclude that

$T=T_{c}+T_{w R}+T_{c}=\frac{1}{2} m\left(\dot{x}_{a}^{2}+\dot{y}_{a}^{2}\right)-$
$m_{c} d \dot{\theta}\left(\dot{y}_{a} \cos \theta-\dot{x}_{a} \sin \theta\right)+\frac{1}{2} I_{w}\left(\dot{\varphi}_{R}^{2}+\dot{\varphi}_{L}^{2}\right)+\frac{1}{2} I \dot{\theta}^{2}$

where $m=m_{c}+2 m_{w}$ is the total mass of AV and

$I=I_{c}+m_{c} d^{2}+2 m_{w} L^{2}+2 I_{m}$

is the total equivalent moment of inertia. Since during $2 \mathrm{D}$ movements $\Pi=$ const, the dynamic model of the nonholonomic Euler-Lagrange system 20 becomes

$\frac{d}{d t} \frac{\partial T}{\partial \dot{q}}-\frac{\partial T}{\partial q}=Q_{\text {nonpot }}+\Lambda^{\top}(q) \lambda$

or, in the open format,

$M(q) \ddot{q}+V(q, \dot{q}) \dot{q}=B(q) \tau-\Lambda^{T}(q) \lambda$

where:

$$
\begin{gathered}
M(q)=\left[\begin{array}{ccccc}
m & 0 & -m d \sin \theta & 0 & 0 \\
0 & m & -m d \cos \theta & 0 & 0 \\
-m d \sin \theta & -m d \cos \theta & I & 0 & 0 \\
0 & 0 & 0 & I_{w} & 0 \\
0 & 0 & 0 & 0 & I_{w}
\end{array}\right] \\
V(q, \dot{q})=\left[\begin{array}{ccccc}
0 & 0-m d \dot{\theta} \cos \theta & 0 & 0 \\
0 & 0 & -m d \dot{\theta} \sin \theta & 0 & 0 \\
0 & 0 & 0 & 0 & 0 \\
0 & 0 & 0 & 0 & 0 \\
0 & 0 & 0 & 0 & 0
\end{array}\right], \\
Q_{\text {nonpot }}=B \tau+\mathcal{F}(q, \dot{q}), B=\left[\begin{array}{ll}
1 & 0 \\
0 & 1 \\
0 & 0 \\
0 & 0 \\
0 & 0
\end{array}\right]
\end{gathered}
$$

Here $\mathcal{F}(q, \dot{q})$ is the centripetal and Coriolis forces, $\tau$ is the control action vector (torques of the right and left motors).

Following 11], let us consider the kinematic relation

$\dot{q}=S \dot{\varphi}, \varphi=\left(\begin{array}{c}\varphi_{R} \\ \varphi_{L}\end{array}\right)$

where

$S(q)=\frac{1}{2}\left[\begin{array}{cc}R \cos \theta & R \cos \theta \\ R \sin \theta & R \sin \theta \\ \frac{R}{L} & -\frac{R}{L} \\ 2 & 0 \\ 0 & 2\end{array}\right]$

It can be verified that the matrix $S(q)$ (24) acts in the null space of the constraint matrix $\Lambda^{T}(q)$, that is,

$\Lambda(q) S(q)=0, S^{\top}(q) \Lambda^{\top}(q)=0$ and therefore, differentiation of 23$]$, leads to

$\ddot{q}=\dot{S}(q) \dot{\varphi}+S(q) \ddot{\varphi}$

Substitution 26 into 22 gives

$$
\begin{aligned}
M(q)(\dot{S}(q) \dot{\varphi} & +S(q) \ddot{\varphi})+V(q, \dot{q}) S(q) \dot{\varphi}=B \tau \\
& +\mathcal{F}(q, \dot{q})-\Lambda^{T}(q) \lambda
\end{aligned}
$$

Multiplying both sides of 27) from left by $S^{\top}(q)$ and using the property 25 , we finally get

$D(q) \ddot{\varphi}+\bar{V}(q, \dot{q}) \dot{\varphi}=\bar{B} \tau+\overline{\mathcal{F}}(q, \dot{q})$

where

$$
\begin{gathered}
D(q)=S^{\boldsymbol{\top}}(q) M(q) S(q), \\
\bar{V}(q, \dot{q})=S^{\boldsymbol{\top}}(q)[M(q) \dot{S}(q)+V(q, \dot{q}) S(q)] \\
\bar{B}(q)=S^{\boldsymbol{\top}}(q) B=\left[\begin{array}{ll}
1 & 0 \\
0 & 1
\end{array}\right], \overline{\mathcal{F}}(q, \dot{q})=S^{\boldsymbol{\top}}(q) \mathcal{F}(q, \dot{q})
\end{gathered}
$$

\section{Assumptions and problem formulation}

Main assumptions

Consider the following assumption hold through the entire work:

1. Vectors $q_{t}, \dot{q}_{t}$ are phisically measured for all $t \geq 0$.

2. Inertia Matrix $D\left(q_{t}\right)$, which can contain unknown terms a priori, it is strictly positive defined and bounded, i.e.,

$$
\left(0<D_{-} I_{n x n} \leq D(q) \leq D_{+} I_{n \times n}\right)
$$

3. The functions $\mathcal{F}$ that defines centripetal, Coriolis forces and may include the friction and hysteresis effects description is assumed to be unknown, but bounded:

$\left\|\mathcal{F}\left(q_{t}, \dot{q}_{t}\right)\right\| \leq F_{+}$

( $F_{+}$is supposed to be known). 
5.1 Cost function with penalty terms

Consider the distance $\delta_{t}$ between the current AV position $q_{t}$ and a desired final point $q^{*}$ :

$\delta_{t}:=q_{t}-q^{*}$

Let the cost functional be convex (not necessarily strictly convex) $F: \mathbb{R}^{n} \longrightarrow \mathbb{R}$, which defines the quality of the actions of the control $\tau_{t}$. The following expression considers the class of convex functions to optimize:

$F(\delta)=\sum_{i=1}^{n}\left|\delta_{i}\right|+\sum_{j=1}^{m} \frac{\mu_{j}}{\left[g_{j}(\delta)\right]_{+}+\epsilon_{j}}$

where:

- $g_{j}(\delta)$ - describes the forbidden area

$g_{j}(\delta) \leq 0$

- the operator $[\cdot]_{+}: \mathbb{R} \rightarrow \mathbb{R}$ is defined as

$[z]_{+}:=\left\{\begin{array}{l}z \text { if } z \geq 0 \\ 0 \text { if } z<0\end{array}\right.$,

- $\mu_{j}, \epsilon_{0}$ are positive parameters.

\subsection{Problem formulation}

Problem 1 Under the assumptions 1-3 above, we need to find a control strategy $\tau$ which provides the functional convergence of the cost $F\left(\delta_{t}\right)$ to its minimum value $F^{*}$, in the presence of uncertainties $\mathcal{F}\left(q_{t}, \dot{q}_{t}\right)$, that is,

$F\left(\delta_{t}\right) \underset{t \longrightarrow \infty}{\longrightarrow} \quad \inf _{\delta \in \mathbb{R}^{n}: g_{j}(\delta)>0, j=1, \ldots, m} F(\delta)=F^{*}$

\section{Desired dynamics and its properties}

6.1 Auxilary sliding variable $s_{t}$

Define the vector function $s_{t} \in \mathbb{R}^{n}$, which from now on and throughout this work will be referred to as "sliding variable":

$$
\begin{gathered}
s_{t}=\dot{\delta}_{t}+\frac{\delta_{t}+\eta}{t+\theta}+\tilde{G}_{t}, \eta \in \mathbb{R}^{n} \\
\tilde{G}_{t}:=\frac{1}{t+\theta} \int_{\tau=t_{0}}^{t} a\left(\delta_{\tau}\right) d \tau, \theta>0 \\
\quad a\left(\delta_{\tau}\right) \in \partial F\left(\delta_{\tau}\right)
\end{gathered}
$$

Here $\delta_{t} \in \mathbb{R}^{n}$ is defined in 32 and $\tilde{G}_{t}$ is the averaged subgradient of the function $F\left(q_{t}\right)$ 35.

Remark 1 Note that the sliding variable $s_{t}$ contains the integral term which is physically measurable.

\subsubsection{Desired dynamic}

The sliding variable $s_{t}$ is associated with following system of ODE:

$$
\begin{gathered}
\dot{\zeta}_{t}=-a\left(\delta_{t}\right), \zeta_{t_{0}}=0, \quad \delta_{t t_{0}} \text { is given } \\
(t+\theta) \dot{\delta}_{t}+\delta_{t}+\eta=\zeta_{t}, \quad t \geq t_{0} \geq 0
\end{gathered}
$$

$t_{0}$ is the moment when the desired dynamics may begin. $\}$

which is refered here to as a "desired dynamic". It is evident that

$(t+\theta) s_{t}=(t+\theta) \dot{\delta}_{t}+\delta_{t}+\eta=\zeta_{t}$,

and therefore the desired dynamics (37) corresponds exactly to the situation when the sliding variable $s_{t}$ is equal to zero for all $t \geq t_{0}$ :

$s_{t}=\dot{s}_{t}=0$.

Below we will show why the dynamic (37) is called a desired.

Lemma 1 (Functional convergence in the desired regime.) For the variable $\delta_{t}$ satisfying the ideal dynamics (38), with any $\theta>0$ and $\eta$, for all $t \geq t_{0} \geq 0$ the following inequality is guaranteed:

$F\left(\delta_{t}\right)-F^{*} \leq \frac{\Phi_{t_{0}}}{t+\theta} \underset{t \rightarrow \infty}{\rightarrow} 0$

where

$\Phi_{t_{0}}=\Phi\left(\delta_{t_{0}}, \theta, \eta\right):=\left(t_{0}+\theta\right)\left[F\left(\delta_{t_{0}}\right)-F^{*}\right]$

$$
+\frac{1}{2}\left\|\delta^{*}-\eta\right\|^{2}
$$

Proof Defining $\mu_{t}:=t+\theta$ and

$\delta^{*}:=\arg \inf _{\delta \in \mathbb{R}^{n}: g_{j}(\delta)>0, j=1, \ldots, m} F(\delta)$

we have

$$
\begin{gathered}
\frac{d}{d t}\left[\frac{1}{2}\left\|\zeta_{t}\right\|^{2}-\zeta_{t}^{\top} \delta^{*}\right]=\dot{\zeta}_{t}^{\top}\left(\zeta_{t}-\delta^{*}\right)= \\
-a^{\top}\left(\delta_{t}\right)\left[\mu_{t} \dot{\delta}_{t}+\delta_{t}+\eta-\delta^{*}\right]= \\
-a^{\top}\left(\delta_{t}\right)\left(\delta_{t}-\delta^{*}\right)-a^{\top}\left(\delta_{t}\right)\left(\mu_{t} \dot{\delta}_{t}+\eta\right) .
\end{gathered}
$$

Using the inequality (see chapter 23 in $[14$ )

$\left(\delta-\delta^{*}\right)^{T} a(\delta) \geq F(\delta)-F^{*}$

valid for convex (not obligatory stongly convex) functions in the first term on the right side, and applying the identity. 
$a^{T}\left(\delta_{t}\right) \dot{\delta}_{t}=\frac{d}{d t}\left[F\left(\delta_{t}\right)-F^{*}\right]$

we get

$$
\begin{gathered}
\frac{d}{d t}\left[\frac{1}{2}\left\|\zeta_{t}\right\|^{2}-\zeta_{t}^{T} \delta^{*}\right] \leq-\left[F\left(\delta_{t}\right)-F^{*}\right] \\
-\mu_{t} \frac{d}{d t}\left[F\left(\delta_{t}\right)-F^{*}\right]-a^{T}\left(\delta_{t}\right) \eta
\end{gathered}
$$

Then, integrating the last inequality in the interval $\left[t_{0}, t\right]$ and applying the formula of integration by parts, we derive

$$
\begin{gathered}
\int_{\tau=t_{0}}^{t}\left[F\left(\delta_{\tau}\right)-F^{*}\right] d \tau \leq \frac{1}{2}\left(\left\|\zeta_{t_{0}}\right\|^{2}-\left\|\zeta_{t}\right\|^{2}\right)+ \\
\left(\zeta_{t}-\zeta_{t_{0}}\right)^{T} \delta^{*}-\left(\mu_{t}\left[F\left(\delta_{t}\right)-F^{*}\right]\right)_{t_{0}}^{t}+ \\
\int_{\tau=t_{0}}^{t}\left[F\left(\delta_{\tau}\right)-F^{*}\right] \dot{\mu}_{\tau} d \tau-\left[\int_{\tau=t_{0}}^{t} a^{T}\left(\delta_{\tau}\right) d \tau\right] \eta .
\end{gathered}
$$

Since $\dot{\mu}_{\tau}=1$, the above inequality becomes

$$
\begin{gathered}
\mu_{t}\left[F\left(\delta_{t}\right)-F^{*}\right] \leq \mu_{t_{0}}\left[F\left(\delta_{t_{0}}\right)-F^{*}\right] \\
\frac{1}{2}\left(\left\|\zeta_{t_{0}}\right\|^{2}-\left\|\zeta_{t}\right\|^{2}\right)+\left(\zeta_{t}-\zeta_{t_{0}}\right)^{\top} \delta^{*}+\zeta_{t}^{\top} \eta= \\
\left(t_{0}+\theta\right)\left[F\left(\delta_{t_{0}}\right)-F^{*}\right]+\left(\frac{1}{2}\left\|\zeta_{t_{0}}\right\|^{2}-\zeta_{t_{0}}^{\top} \delta^{*}\right)+ \\
\frac{1}{2}\left\|\delta^{*}-\eta\right\|^{2}-\frac{1}{2} \underbrace{\left[\left\|\zeta_{t}\right\|^{2}-2 \zeta_{t}^{\top}\left(\delta^{*}-\eta\right)+\left\|\delta^{*}-\eta\right\|^{2}\right]}_{\left\|\zeta_{t}-\left(\delta^{*}-\eta\right)\right\|^{2}} \\
\leq\left(t_{0}+\theta\right)\left[F\left(\delta_{t_{0}}\right)-F^{*}\right]-\frac{1}{2}\left\|\zeta_{t}-\left(\delta^{*}-\eta\right)\right\|^{2}+ \\
\left(\frac{1}{2}\left\|\zeta_{t_{0}}\right\|^{2}-\zeta_{t_{0}}^{T} \delta^{*}\right)+\frac{1}{2}\left\|\delta^{*}-\eta\right\|^{2} \leq \Phi_{t_{0}},
\end{gathered}
$$

from which we obtain 40 . Lemma is proved.

Remark 2 The parameter $\eta$ will be chosen below in such a way that the desired optimization regime starts from the beginning of the process, when, $t_{0}=0$.

\section{ISM robust controller}

7.1 Main theorem on robust controller

Theorem 1 Under assumptions 1-3 the ISM robust controller

$$
\begin{gathered}
\tau_{t}=D\left(q_{t}\right) S^{\boldsymbol{\top}}\left(q_{t}\right)\left[S\left(q_{t}\right) S^{\boldsymbol{\top}}\left(q_{t}\right)+\rho I_{5 \times 5}\right]^{-1} \\
{\left[-k_{t} \operatorname{SIGN}\left(s_{t}\right)+u_{c o m p, t}\right]} \\
u_{c o m p, t}=-\left(I_{5 \times 5}+\rho\left[S\left(q_{t}\right) S^{\boldsymbol{\top}}\left(q_{t}\right)+\rho I_{5 \times 5}\right]^{-1}\right)^{-1} p_{t}^{\text {reali }} \\
, \rho>0 \\
k_{t}=\left\|S\left(q_{t}\right) D^{-1}\left(q_{t}\right) S^{\boldsymbol{\top}}\left(q_{t}\right)\right\| F^{+}+\rho_{0}, \rho_{0}>0
\end{gathered}
$$

where

$$
\begin{gathered}
p_{t}^{\text {reali }}:=\dot{S}(q) \dot{\varphi}-S(q) D^{-1}(q) \bar{V}(q, \dot{q}) \dot{\varphi} \\
+\frac{1}{t+\theta}\left(\dot{\delta}_{t}-\frac{\delta_{t}+\eta}{t+\theta}-\tilde{G}_{t}+a\left(\delta_{t}\right)\right)
\end{gathered}
$$

with

$$
\eta=-\theta \delta_{2,0}-\delta_{1,0}
$$

guarantees the functional convergence (39) from the beginning of the process $\left(t_{0}=0\right)$.

Proof In view of (29) we may conclude that the matrix $D(q)$ is positive definite and, hence, is invertible. Then by the relations (26) and (28) we obtain

$$
\begin{gathered}
\delta_{t}:=q_{t}-q^{*}, \dot{\delta}_{t}=\dot{q}_{t} \\
\ddot{\delta}_{t}=\ddot{q}_{t}=\dot{S}(q) \dot{\varphi}+S(q) \ddot{\varphi}= \\
\dot{S}(q) \dot{\varphi}-S(q) D^{-1}(q) \bar{V}(q, \dot{q}) \dot{\varphi}+ \\
S(q) D^{-1}(q) \tau+S(q) D^{-1}(q) \overline{\mathcal{F}}(q, \dot{q})
\end{gathered}
$$

For the Lyapunov function $V(s)=\frac{1}{2} s^{\top} s$ we have

$$
\begin{gathered}
\dot{V}\left(s_{t}\right)=s_{t}^{\top} \dot{s}_{t}= \\
s_{t}^{\top}\left(\ddot{\delta}_{t}+\frac{\dot{\delta}_{t}}{t+\theta}-\frac{\delta_{t}+\eta}{(t+\theta)^{2}}-\frac{1}{t+\theta} \tilde{G}_{t}+\frac{1}{t+\theta} a\left(\delta_{t}\right)\right)= \\
s_{t}^{\top}\left(\dot{S}(q) \dot{\varphi}-S(q) D^{-1}(q) \bar{V}(q, \dot{q}) \dot{\varphi}\right. \\
+S(q) D^{-1}(q) \tau+S(q) D^{-1}(q) \overline{\mathcal{F}}(q, \dot{q})+ \\
\left.\frac{1}{t+\theta}\left(\dot{\delta}_{t}-\frac{\delta_{t}+\eta}{t+\theta}-\tilde{G}_{t}+a\left(\delta_{t}\right)\right)\right)= \\
s_{t}^{\top} p_{t}^{\text {reali }}+s_{t}^{\top} S(q) D^{-1}(q) \tau+s_{t}^{\top} S(q) D^{-1}(q) \overline{\mathcal{F}}(q, \dot{q})
\end{gathered}
$$

Selecting $\tau$ as in (43) for the second term in (46) we have

$$
\begin{aligned}
& s_{t}^{T} S(q) D^{-1}(q) \tau= \\
& s_{t}^{T} S(q) S^{\boldsymbol{\top}}(q)\left[S(q) S^{\boldsymbol{\top}}(q)+\rho I_{5 \times 5}\right]^{-1} \\
& {\left[-k_{t} \mathrm{SIGN}\left(s_{t}\right)+u_{c o m p, t}\right]} \\
& =s_{t}^{T}\left[S(q) S^{\top}(q)+\rho I_{5 \times 5}-\rho I_{5 \times 5}\right] \\
& {\left[S(q) S^{\top}(q)+\rho I_{5 \times 5}\right]^{-1}\left[-k_{t} \operatorname{SIGN}\left(s_{t}\right)+u_{c o m p, t}\right]} \\
& =s_{t}^{T}\left[S(q) S^{\top}(q)+\rho I_{5 \times 5}-\rho I_{5 \times 5}\right] \\
& {\left[S(q) S^{\top}(q)+\rho I_{5 \times 5}\right]^{-1}} \\
& {\left[-k_{t} \operatorname{SIGN}\left(s_{t}\right)+u_{c o m p, t}\right]} \\
& =s_{t}^{T}\left[S(q) S^{\top}(q)+\rho I_{5 \times 5}-\rho I_{5 \times 5}\right] \\
& +s_{t}^{T}\left(I_{5 \times 5}+\rho\left[S(q) S^{\top}(q)+\rho I_{5 \times 5}\right]^{-1}\right) u_{c o m p, t}
\end{aligned}
$$


Then (46) becomes

$$
\begin{aligned}
& \dot{V}\left(s_{t}\right)=-k_{t} s_{t}^{T} \operatorname{SIGN}\left(s_{t}\right) \\
& +s_{t}^{T}\left(I_{5 \times 5}+\rho\left[S(q) S^{\top}(q)+\rho I_{5 \times 5}\right]^{-1}\right) u_{c o m p, t}+ \\
& s_{t}^{T} p_{t}^{r e a l i}+s_{t}^{T} S(q) D^{-1}(q) \overline{\mathcal{F}}(q, \dot{q}) \\
& \leq-k_{t} \sum_{i=1}^{n}\left|s_{i, t}\right|+ \\
& s_{t}^{T}\left[\left(I_{5 \times 5}+\rho\left[S(q) S^{\top}(q)+\rho I_{5 \times 5}\right]^{-1}\right) u_{c o m p, t}+p_{t}^{r e a l i}\right]+\ddot{\delta}=h_{0}(q, \dot{q})+\tilde{B}(q) \tau \\
& \left\|s_{t}\right\|\left\|S(q) D^{-1}(q) S^{\top}(q)\right\| \underbrace{\|\mathcal{F}(q, \dot{q})\|}_{\leq F^{+}}
\end{aligned}
$$

Taking into account that

$\sum_{i=1}^{n}\left|s_{i, t}\right| \geq\left\|s_{t}\right\|$

and selecting the compensating control $u_{\text {comp }}$ and $k_{t}$ as in (43) from (47) we derive

$\dot{V}\left(s_{t}\right) \leq-\rho_{0}\left\|s_{t}\right\|=-\sqrt{2} \rho_{0} \sqrt{V\left(s_{t}\right)}$

implying

$2\left(\sqrt{V\left(s_{t}\right)}-\sqrt{V\left(s_{t_{0}}\right)}\right) \leq-\sqrt{2} \rho_{0} t$

and

$0 \leq \sqrt{V\left(s_{t}\right)} \leq \sqrt{V\left(s_{t_{0}}\right)}-\frac{\rho_{0}}{\sqrt{2}} t$

which leads to the conclusion that for al $l t \geq t_{\text {reach }}:=$ $\frac{1}{\rho_{0}} \sqrt{2 V\left(s_{t_{0}}\right)}=\frac{\left\|s_{t_{0}}\right\|}{\rho_{0}}$ we have that $V\left(s_{t}\right)=0$ and $s_{t}=$ 0. Since by 36

$s_{t_{0}}=\dot{\delta}_{t_{0}}+\frac{\delta_{t_{0}}+\eta}{t_{0}+\theta}$

taking $\eta$ as in 45 , we obtain that $s_{0}=\delta_{2,0}+\frac{\delta_{1,0}+\eta}{\theta}=$ 0 and $t_{\text {reach }}=\frac{\left\|s_{0}\right\|}{\rho_{0}}=0$. The theorem is proven.

\section{2D car model avoiding obstacles}

To show the good performance of the method hereby suggested let us apply the controller (43) to the systems given by equation (22) and the main objective is that the system reaches a desired point $\left(x^{*}, y^{*}\right)$ in $X Y$ - plane despite the presence of several obstacles disposed in the same plane.
8.1 Selected functions and parameters

The dynamic equation 22 of the considered system

Select the cost function $F(\delta)$, which includes also the penalty terms for 3 obstacles (two ellipsoids and one triangle)

$$
\begin{aligned}
& f_{1}=f_{2}=1, f_{i>2}=0, x_{1}^{*}=8, y_{1}^{*}=13, \\
& \chi=0.01 *, \epsilon_{0}=0.5, \theta=0.1 \\
& \overline{\mathcal{F}}(q, \dot{q})=-\theta \underbrace{\left[\begin{array}{lllll}
1 & 1 & 0 & 1 & 1 \\
3 & 2 & 0 & 2 & 2
\end{array}\right]}_{\Theta} \dot{q}=-\theta \Theta \dot{q} \\
& g_{1}(\delta)=\left[\left(\begin{array}{c}
\delta_{1}+x_{1}^{*}-x_{01} \\
\delta_{2}+y_{1}^{*}-y_{01}
\end{array}\right)^{T} A_{1}\left(\begin{array}{c}
\delta_{1}+x_{1}^{*}-x_{01} \\
\delta_{2}+y_{1}^{*}-y_{01}
\end{array}\right)\right. \\
& -1+\chi]_{+} \\
& x_{01}=0, y_{01}=0, \mu_{1}=1, A_{1}=\left[\begin{array}{ll}
0.3 & 0.4 \\
0.4 & 0.9
\end{array}\right] \\
& g_{2}(\delta)=\left[\left(\begin{array}{c}
\delta_{1}+x_{1}^{*}-x_{02} \\
\delta_{2}+y_{1}^{*}-y_{02}
\end{array}\right)^{T} A_{2}\left(\begin{array}{c}
\delta_{1}+x_{1}^{*}-x_{02} \\
\delta_{2}+y_{1}^{*}-y_{02}
\end{array}\right)\right. \\
& -1+\chi]_{+} \\
& x_{02}=14, y_{02}=7, \mu_{2}=1, A_{2}=\left[\begin{array}{cc}
0.9 & 0 \\
0 & 0.3
\end{array}\right] \\
& \begin{array}{c}
X_{\text {triangle }}=\left\{\begin{array}{c}
\delta: \\
g_{\text {gen }, 1}(\delta)=-\delta_{1}-x^{*}-\delta_{2}-y^{*} \\
+5+\chi \leq 0 \wedge g_{g e n, 2}(\delta)=\delta_{1}+x^{*}-4 \\
+\chi \leq 0 \wedge g_{g e n, 3}(\delta)=-\delta_{1}-x^{*}-\delta_{2}-y^{*} \\
+5+\chi \leq 0
\end{array}\right. \\
\varkappa\left(X_{\text {triangle }}\right):=\sum_{i=1}^{3}\left[g_{\text {gen }, i}(\delta)\right]_{+}^{2}
\end{array}
\end{aligned}
$$


The subgradient has the components

$$
\begin{aligned}
& a(\delta)=\left(\operatorname{sign}\left(\delta_{1}\right) \operatorname{sign}\left(\delta_{2}\right) \quad 0 \quad 0 \quad 0\right)^{\top} \\
& -2 \mu_{1} \frac{\bar{\chi}(g(\delta))}{\left(g(\delta)+\epsilon_{0}\right)^{2}} \\
& \left(\begin{array}{c}
{\left[A\left(\begin{array}{c}
\delta_{1}+x_{1}^{*}-x_{01} \\
\delta_{2}+y_{1}^{*}-y_{01}
\end{array}\right)\right]_{1}} \\
{\left[A\left(\begin{array}{c}
\delta_{1}+x_{1}^{*}-x_{01} \\
\delta_{2}+y_{1}^{*}-y_{01}
\end{array}\right)\right]_{2}} \\
0 \\
0 \\
0
\end{array}\right) \\
& -\mu_{2} \frac{\bar{\chi}\left(g_{d e n}(\delta)\right)}{\left(g_{g e n}(\delta)+\epsilon_{0}\right)^{2}}\left(\begin{array}{c}
\frac{\partial}{\partial \delta_{1}} g_{g e n}(\delta) \\
\frac{\partial}{\partial \delta_{2}} g_{g e n}(\delta) \\
0 \\
0 \\
0
\end{array}\right) \\
& \left(x_{i n i}, y_{i n i}\right)=\left(\begin{array}{l}
-8 \\
-5
\end{array}\right) \\
& \left(x^{*}, y^{*}\right)=\left(\begin{array}{c}
8 \\
13
\end{array}\right)
\end{aligned}
$$

\subsection{Simulation results and discussions}

Finally, the simulation results are depicted in the following figures. The $2 \mathrm{D}$ robot trajectory in plain is shown in Figure (2), which shows how the AV avoids obstacles in order to reach the desired point.

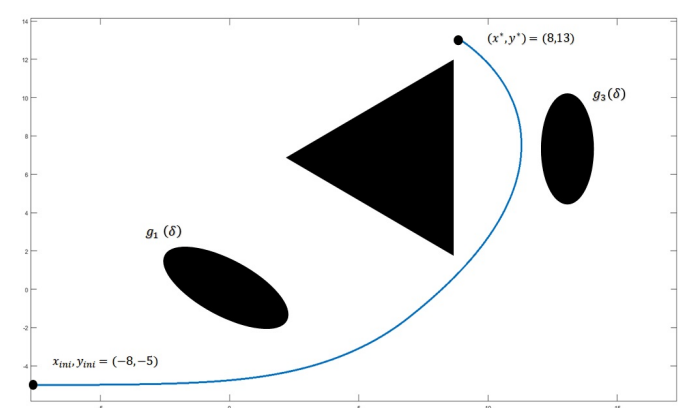

Fig. 2 2D AV trajectory.

In Figure 3, we can see the behaviour of the control signal.

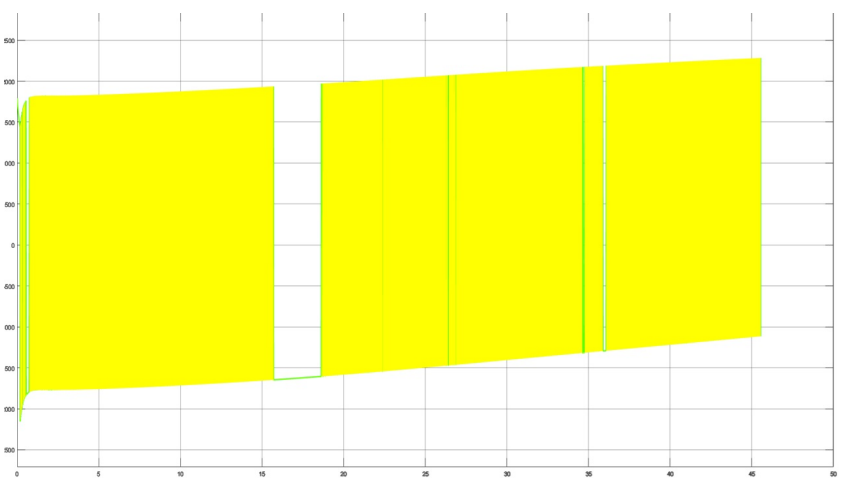

Fig. 3 Control input $\tau_{t}$

Figure 4 shows the behaviour of the Lyapunov function (46) function which is successfully converging to zero as the time increases; a similar behaviour can be seen in Figure 5. because the $s_{t}$ variables converge to zero, meaning the functional convergence is happening and therefore the desired dynamics are obtained, and finally in Figure 6 depicts the cost function converges to its minimum.

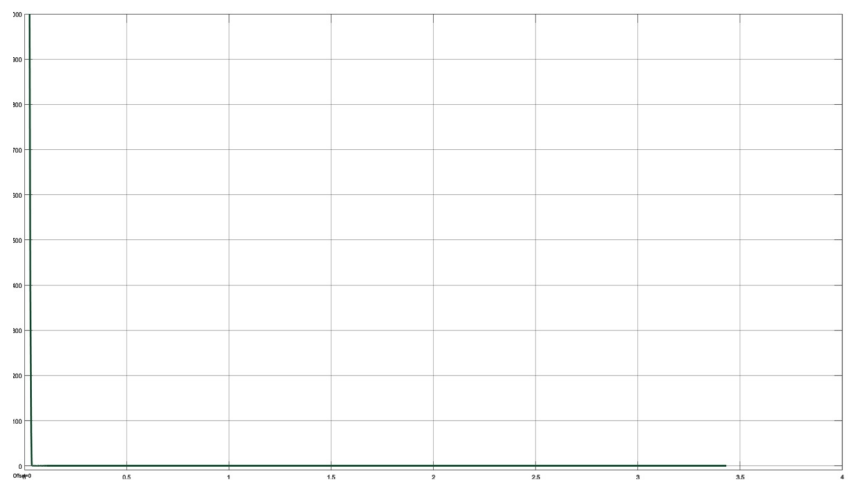

Fig. 4 Lyapunov function converging to zero.

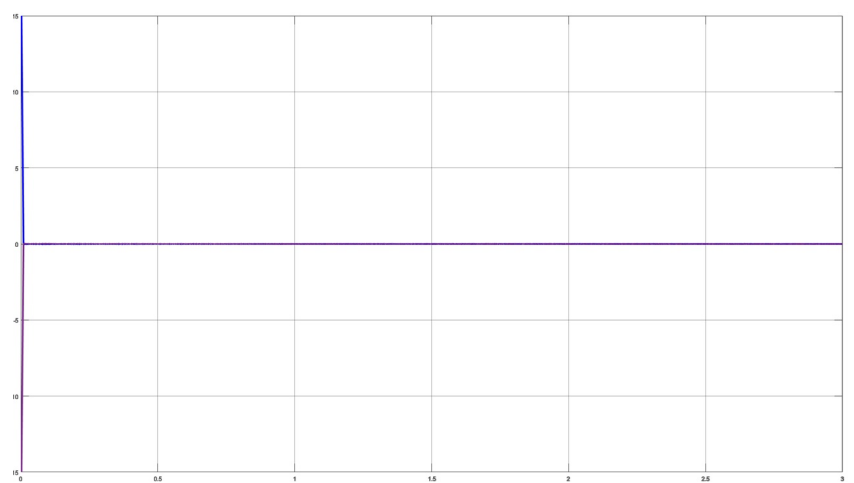

Fig. 5 Sliding variables $s_{t}$ converging to zero. 


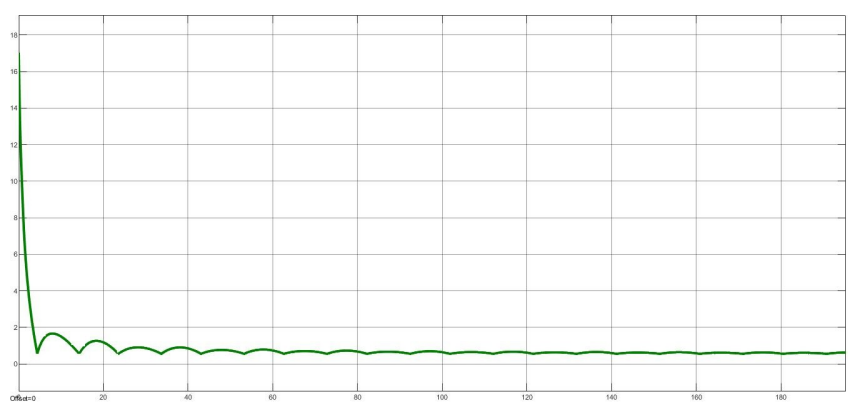

Fig. 6 Cost function reaching its minimum.

\section{Conclusions}

A new robust controller, based on the ASG-version of the convex optimization and which provides obstacles avoidance, is suggested and analyzed. The dynamic of the system includes nonholonomic constrains and admits the presence of some uncertainties. This robust controller is based on Integral Slide Mode control approach along with the sub-gradient technique for optimization of a cost functional, which includes penalty terms allowing the AV Nonholonomic 2D model to avoid the prohibited areas. It is shown that using such structure of the robust controller we may guarantee the desired dynamics from the beginning of the process. Finally, the simulation results show the good working of the suggested method, measuring only the extended state vector and its derivatives, that permits to consider the suggested method as powerful and practical tool for engineering applications.

\section{References}

[1] Q. Z. Shi-gung, Y. Quan-jun, and Z. YinYa-bnig, "Dynamic obstacle-avoiding path planning for robots based on modified potential field method," Springer, 2013. DOI: https ://doi .org/10 .1007/978-3642-39482-9_39.

[2] O. Khatib, "Real time, osbtacle avoidance for manipulators and mobile robots," The International of Robotics Research, pp. 90, 98, 1986. DOI: https: //doi.org/10.1007/s10514-012-9287-y.

[3] T. Mansur Fantazzini and M. T. Arns Steiner, "Vehicle routing problem solved through heuristics procedures: A case study," IEEE LatinAmerica Transactions, vol. 17, no. 5, pp. 858, 864, May 2019. DOI: DOI : 10.1109/TLA . 2017.7910210.

[4] A. S. Huaman Loayza and C. G. Pérez Zuñiga, "Design of a fuzzy sliding mode controller for the autonomous path-following of a quadrotor," IEEE Latin America Transactions, vol. 17, no. 6, pp. 962, 971, Jun. 2019. DOI: DOI:10.1109/TLA. 2019.8896819.

[5] A. Ferrara and V. I. Utkin, "Sliding mode optimization in dynamic lti systems," Journal of Optimization Theory, vol. 115, no. 3, pp. 727740, 2002. DOI: https://doi.org/10.1023/A : 1021267517097 .

[6] A. Poznyak, A. Nazin, and H. Alazki, "Integral sliding mode convex optimization in uncertain lagrangian systems driven by p.m.d.c. motors: Averaged subgradient approach," IEEE Transactions on Automatic Control, Sep. 2021, To be published. DOI: DOI : 10.1109/TAC. 2020.3032088.

[7] Y. Liu and Z. Geng, "Finite-time optimal formation tracking control of vehicles in horizontal plane," Nonlinear Dynamics, vol. 11, no. 76 , pp. 481, 495, Nov. 2013. DOI: https://doi.org/ 10.1007/s11071-013-1141-z.

[8] J. Yuan, H. Chen, F. Sun, and Y. Huang, "Trajectory planning and tracking control for autonomous bicycle robot," Nonlinear Dynamics, vol. 5, no. 78, pp. 421, 431, May 2014. DOI: https://doi.org/ 10.1007/s11071-014-1449-3.

[9] J. Guo, Y. Luo, and K. Li, "Dynamic coordinated control for over-actuated autonomous electric vehicles with nonholonomic constraints via nonsingular terminal sliding mode technique," Nonlinear Dynamics, vol. 4, no. 85, p. 583597 , Mar. 2016. DOI: https://doi.org/10.1007/s11071016-2708-2

[10] H. Ao, F. Gao, and J. Huang, "Barrier lyapunov functions-based fixed-time stabilization of nonholonomic systems with unmatched uncertainties and time-varying output constraints," Nonlinear Dynamics, vol. 99, no. 1, pp. 2835, 2849, Jan. 2020. DOI: doi . org / 10 . 1007 / s11071-01905450-3.

[11] R. Dhaouadi and A. Abu Hatab, "Dynamic modelling of differential-drive mobile robots using lagrange and newton-euler methodologies: A unified framework," Advances in Robotics Automation, 2013. DOI: https : //doi .org/10 .4172/ 2168-9695.1000107.

[12] H. Baruh, Analytical dynamics. McGraw-Hills, 1999, ISBN: 0073659770.

[13] A. M. Bloch, J. Baillieul, P. E. Crouch, and J. E. Marsden, Nonholonomic mechanics and control. Springer-Verlag., 2002. DOI: https://doi .org/ $10.1007 / \mathrm{b} 97376$.

[14] A. Poznyak, Advanced mathematical tools for automatic control engineers: deterministic techniques. Vol 1. Amsterdam, The Netherlands: Elsevier, 2008. DOI: https ://doi .org/10.1134/S0005117909110174. 


\section{Declarations}

Funding

This research work has been financed by the authors without any external support.

Conflicts of interest/Competing interests

Not applicable for this section.

Availability of data and material

Not applicable for this section.

Code availability

Not applicable for this section.

Authors' contributions

- Hector Vargas

- equal idea conceptualization part.

- equal formal analysis part.

- equal investigation part.

- equal methodology plan developer.

- lead software user.

- lead writing -original draft.

- lead writing -edit and review.

- Jesus Meda

- equal investigation part

- equal methodology plan developer.

- supporting help in software use.

- equal supervisor.

- equal validation opinion.

- Supporting writing -edit and review.

- Alexander Poznyak

- equal idea conceptualization part.

- equal formal analysis part.

- supporting investigation part.

- supporting methodology plan developer.

- supporting help in software use.

- equal supervisor.

- equal validation opinion.

- Supporting writing -edit and review.
Consent to participate

Not applicable for this section.

\section{Consent for publication}

The authors authorized the publication of this work. 
Figures

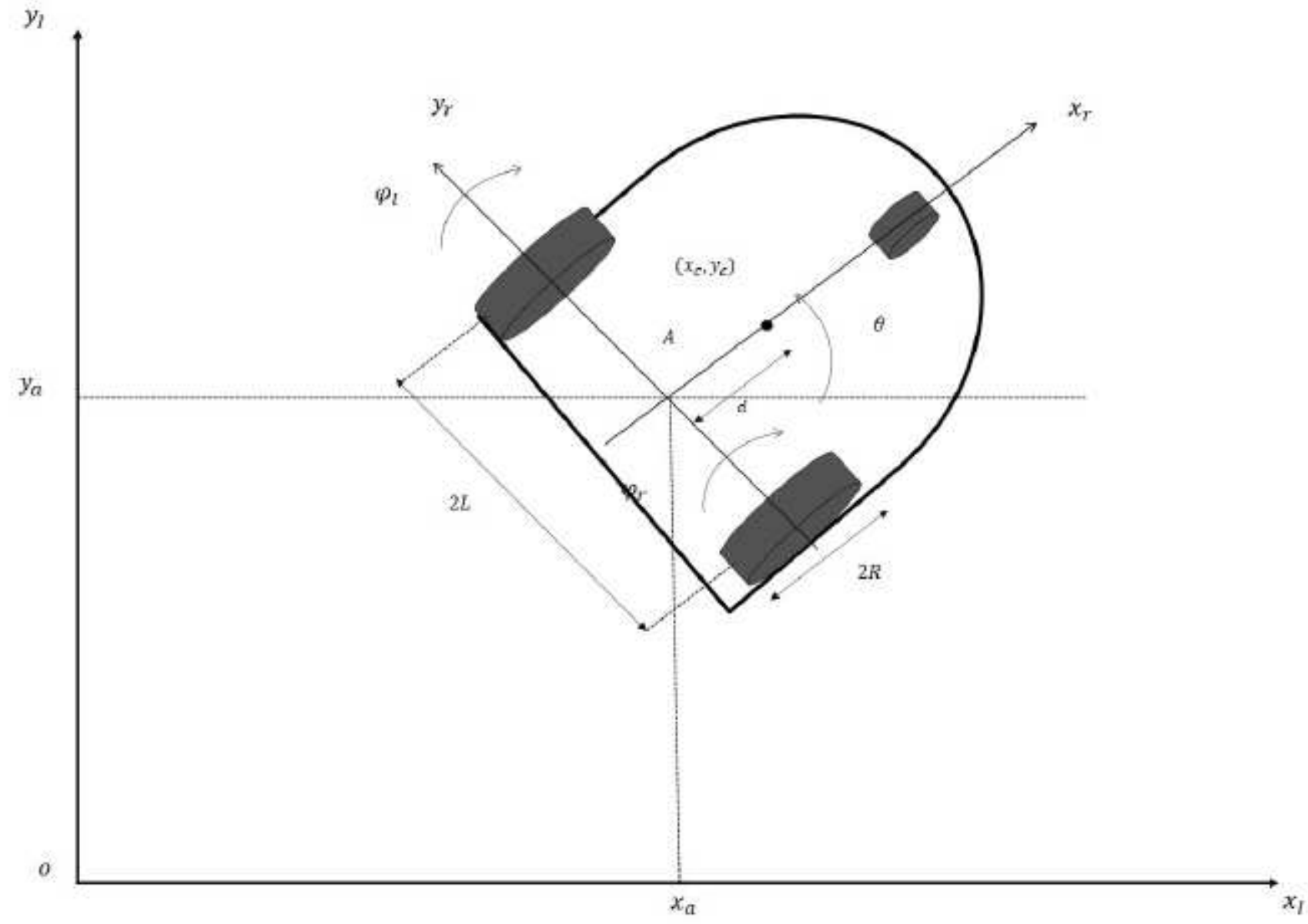

Figure 1

AV description. 


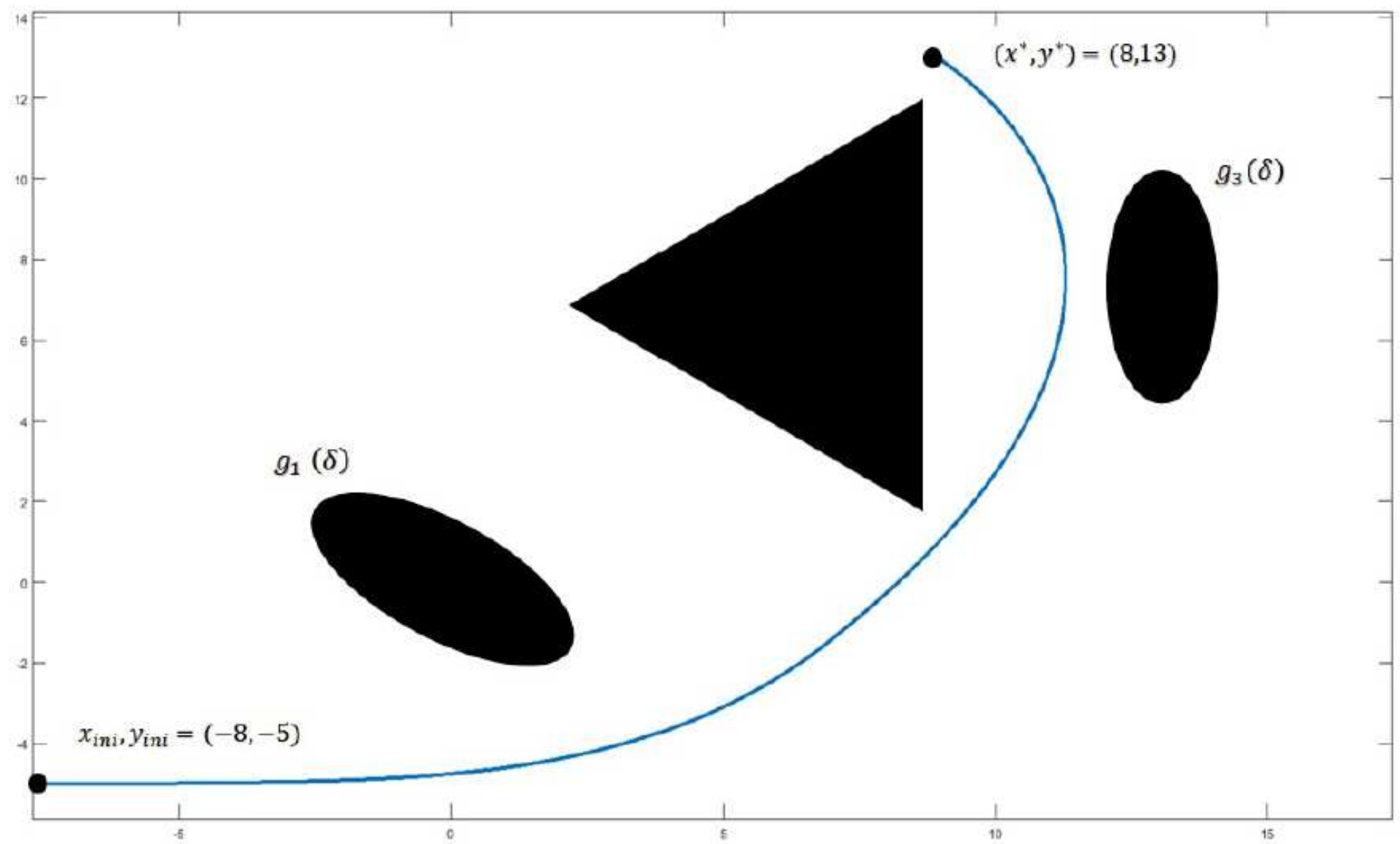

Figure 2

2D AV trajectory.

(50)

(51)

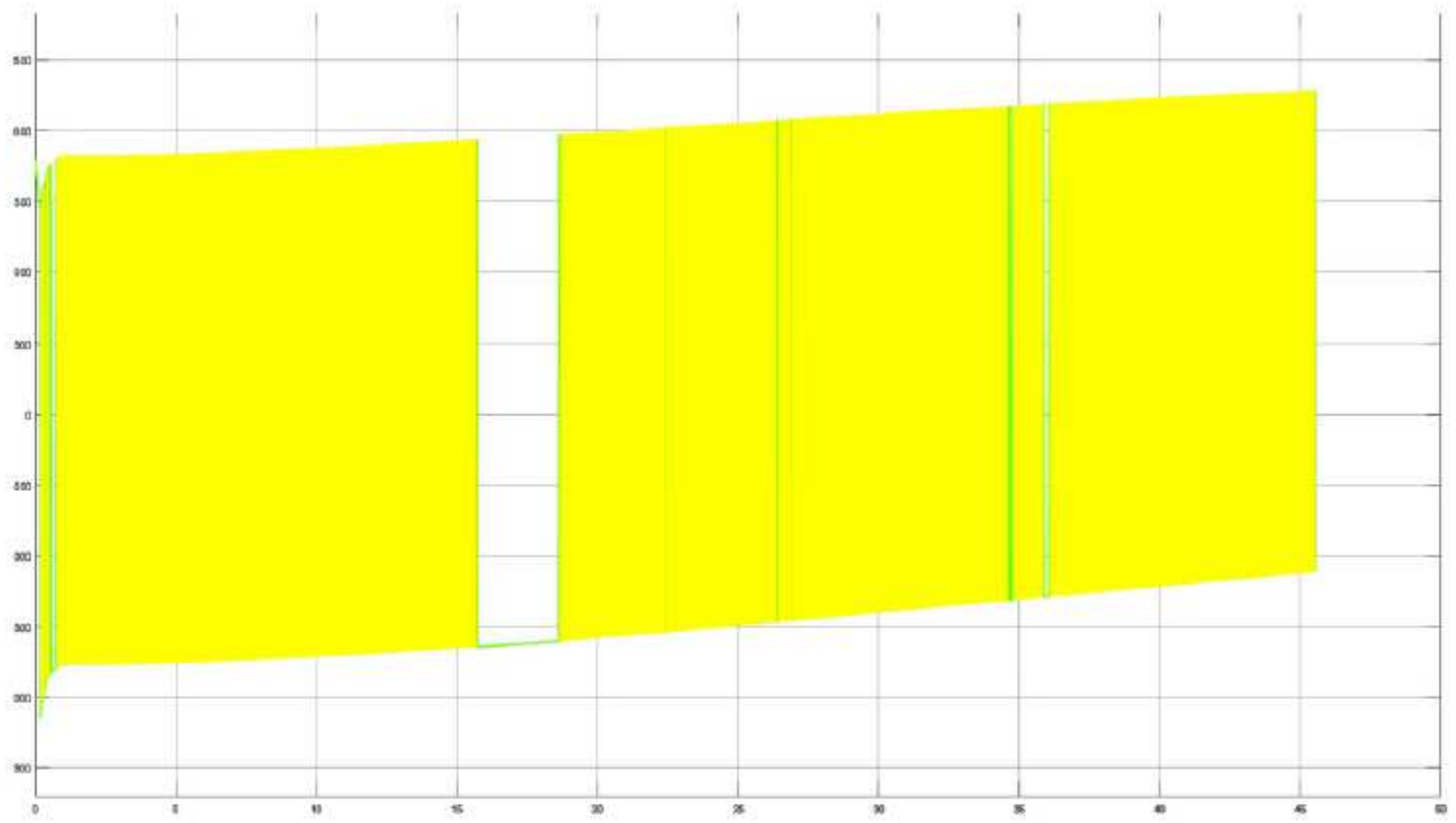

Figure 3 
Control input $\mathrm{Tt}$

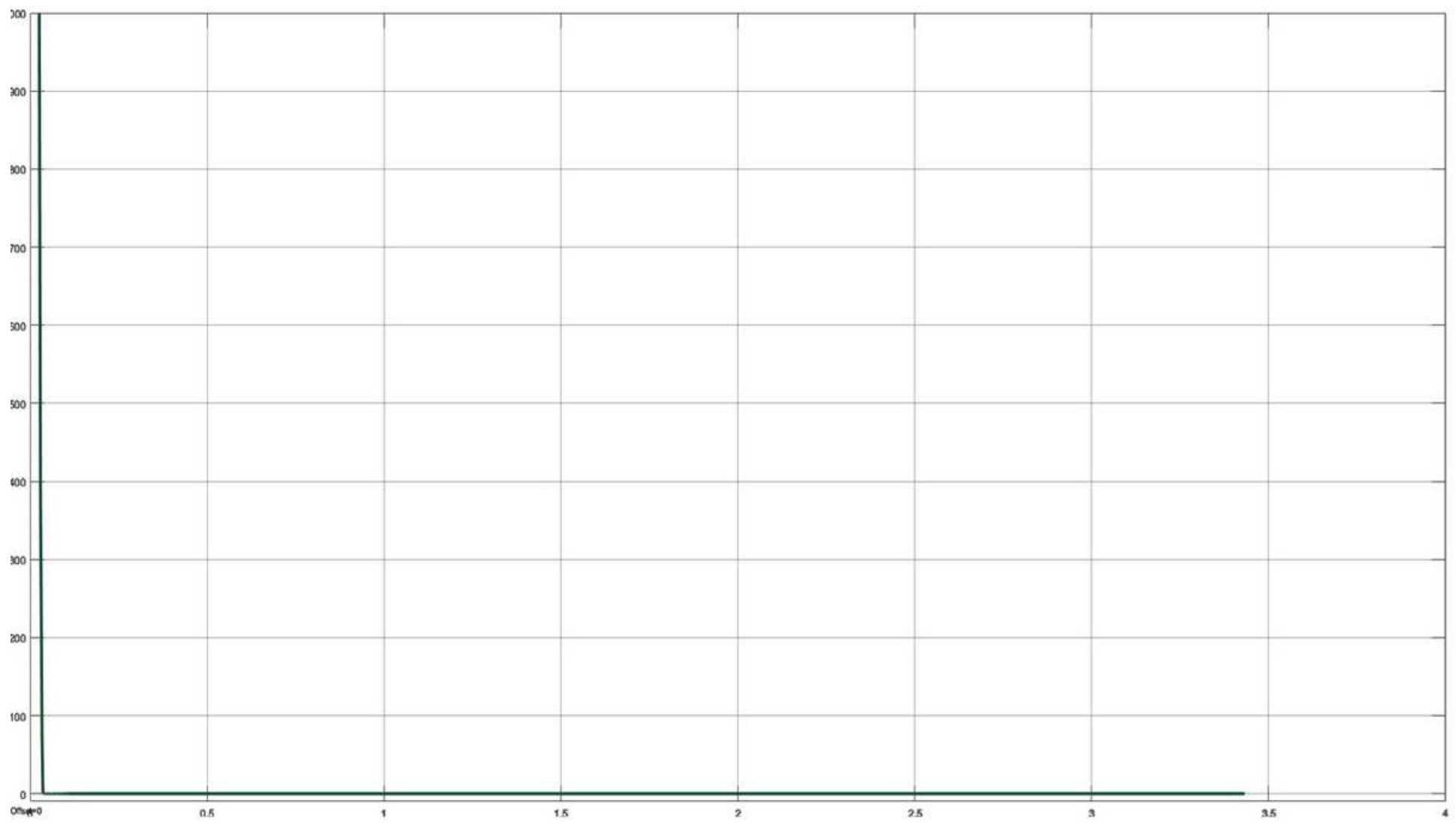

Figure 4

Lyapunov function converging to zero. 


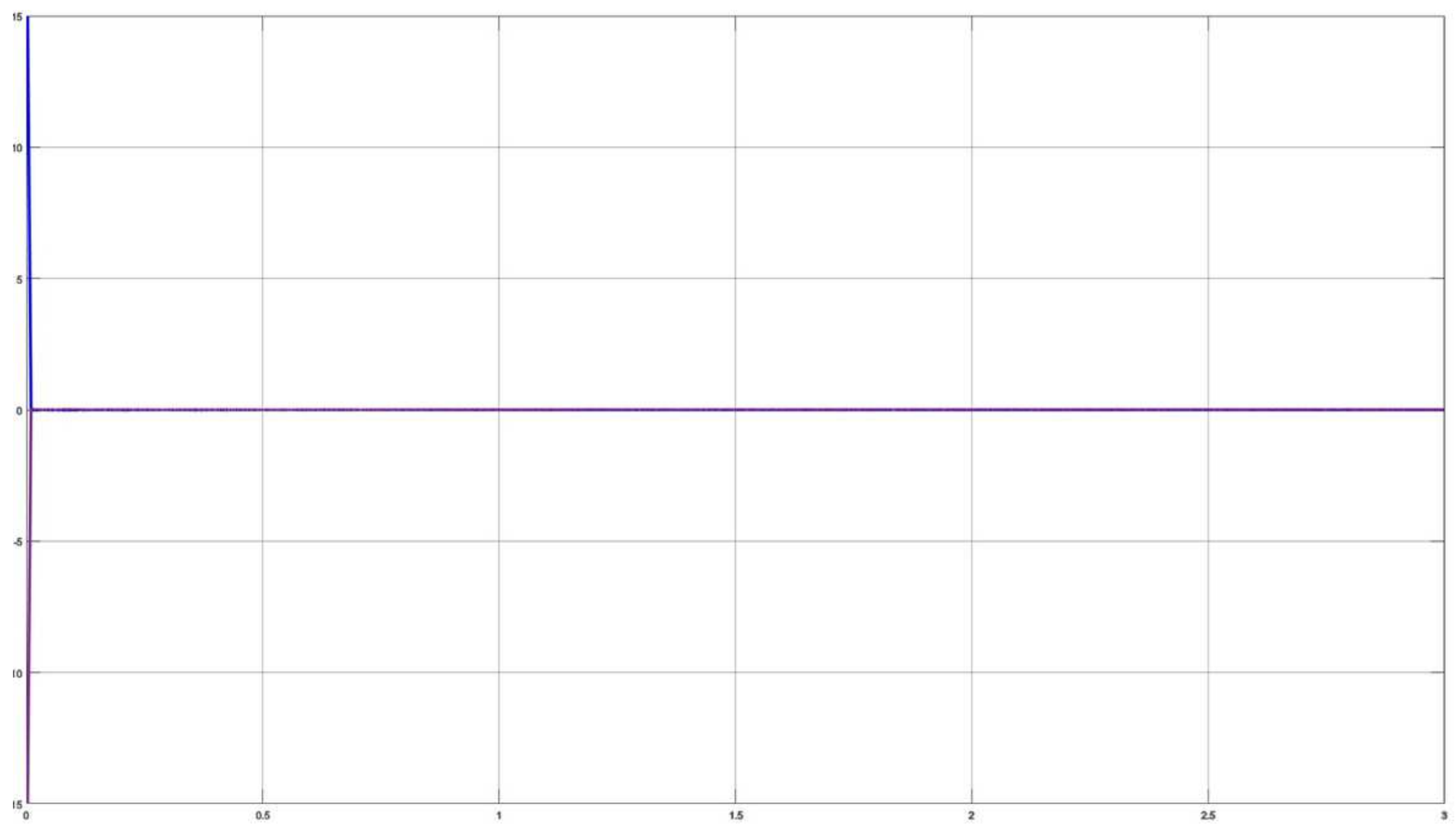

Figure 5

Sliding variables st converging to zero.

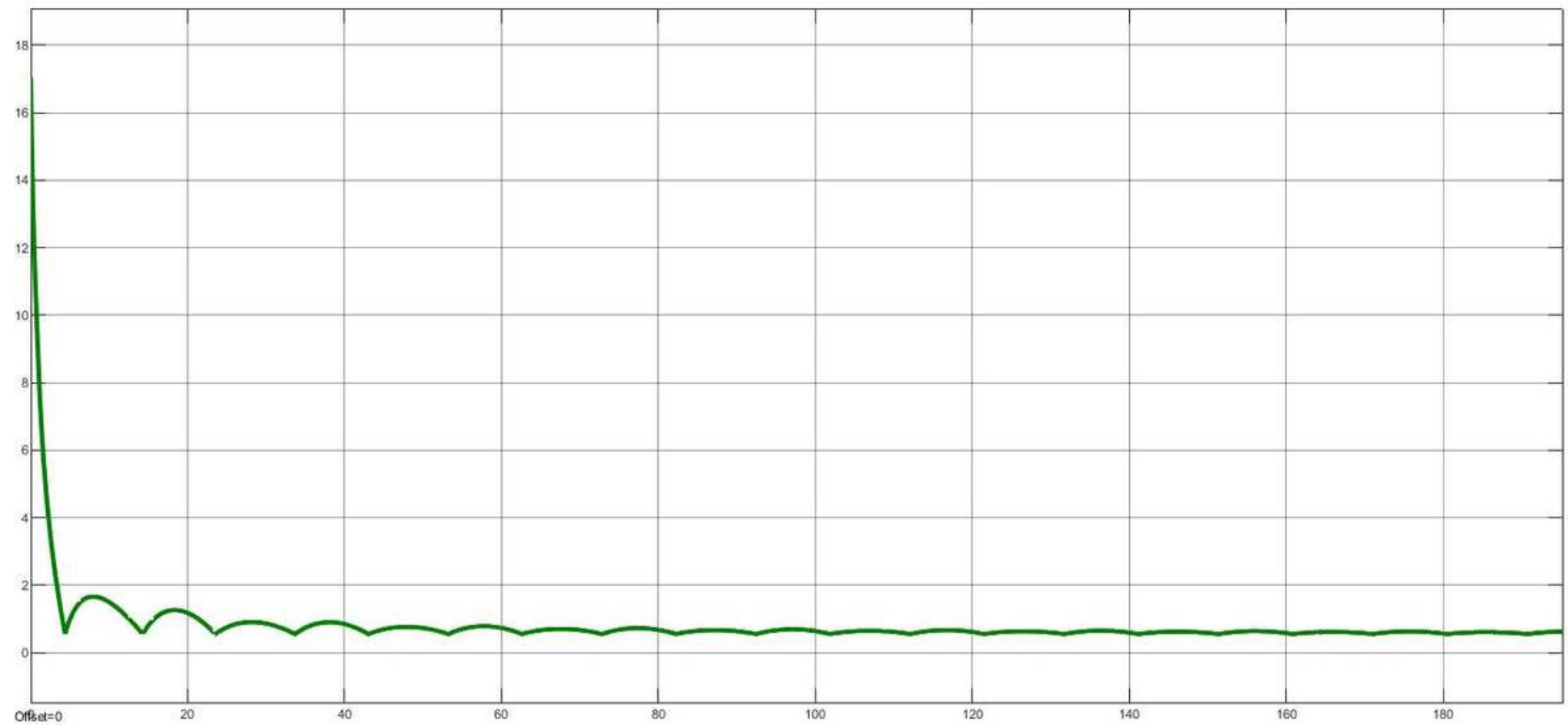

Figure 6

Cost function reaching its minimum. 